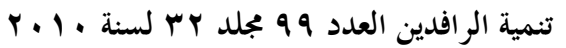

$$
\begin{aligned}
& \text { ص ص[ }
\end{aligned}
$$$$
\text { كلية الإدارة والاقتصاد -جامعة الموصل }
$$

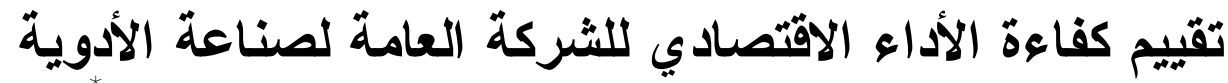

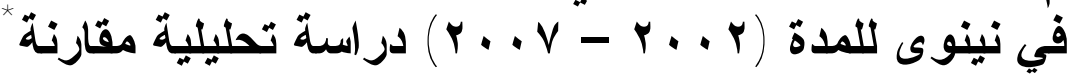

$$
\text { حافظ جاسم عرب المولى }
$$
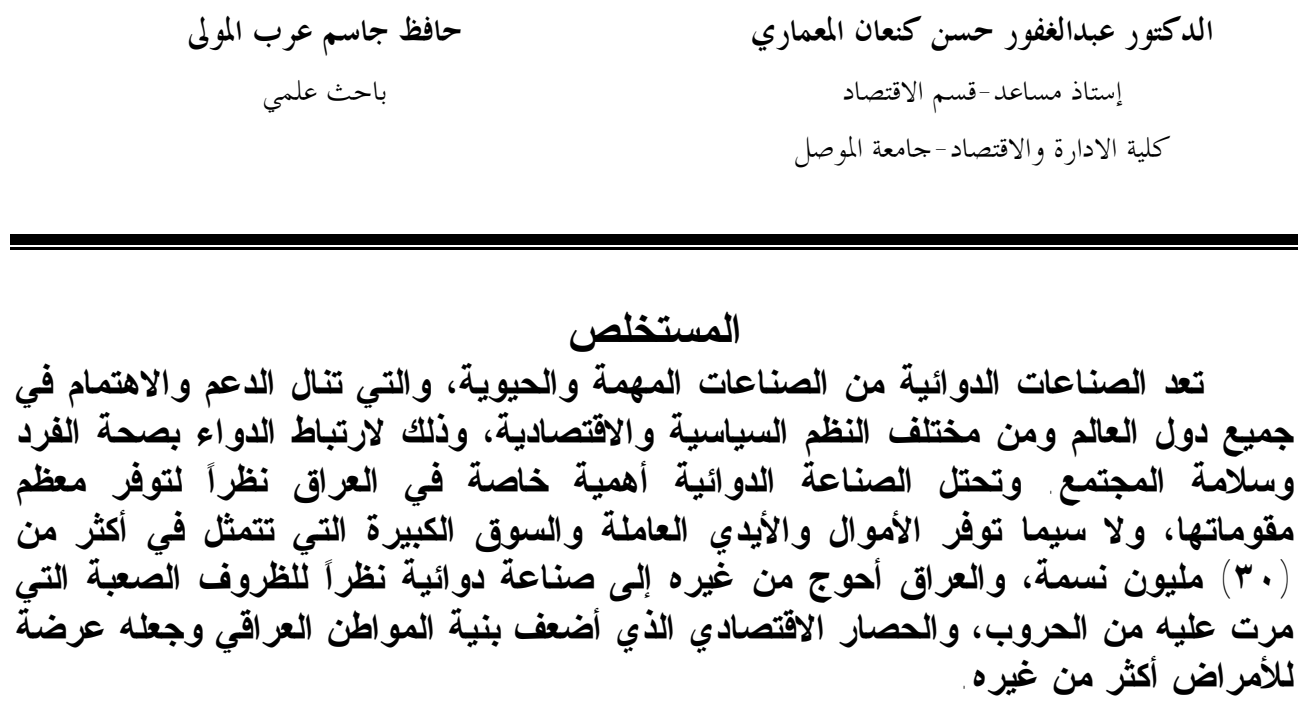

\section{Performance Evaluation of the State Company for Drug Industries in Ninavah (NDI) for the period (2002-2007) Comparative and Analytic Study}

\author{
Abdul-Ghafoor H. Kana'n (PhD) \\ Assistant Professor \\ Department of Economics \\ University of Mosul
}

Jasim A. Al-Moula

Scientific Researcher

Department of Economics

University of Mosul

\footnotetext{
Abstract

The pharmaceutical industries are one of the vital types of industry that receive support and interest by all countries of the world and in different political and economic systems. This is because the medicine is associated with the health of the individual safety of society. The pharmaceutical industry has occupied a drastic importance in Iraq, for the

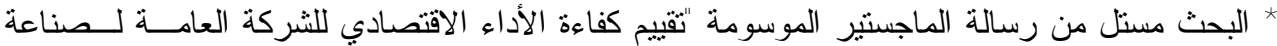

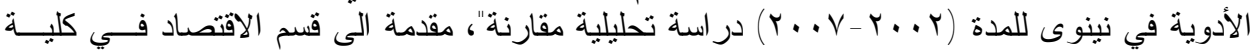

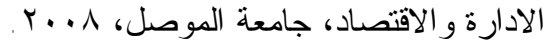

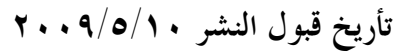

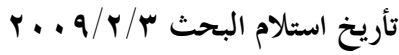


components of this type of industry are highly available, almost funds, manpower and large markets (more than 30 million individual). Iraq nowadays requires these types of industries as for the series wars and embargo that made Iraq citizen fragile for diseases.

تقوم الدول دائما بالبحث عن وسائل لتلبية الحاجات الأساسية لمو اطنيها و إثــــاعها

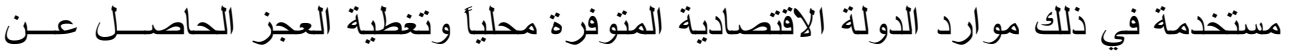

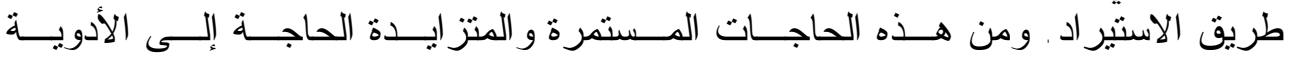

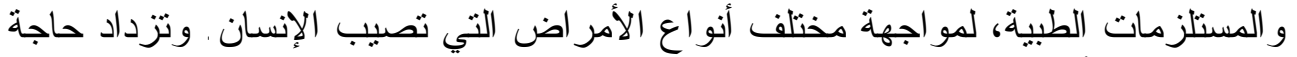

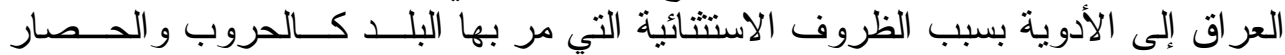

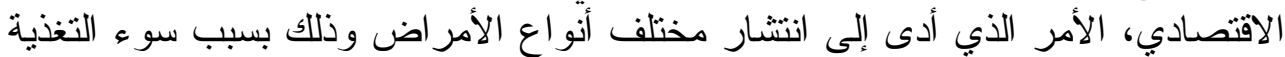

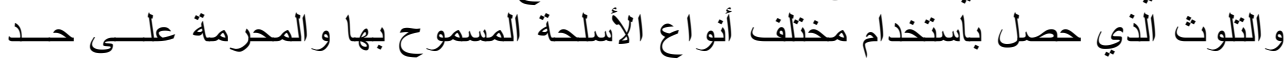

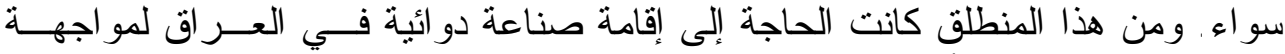

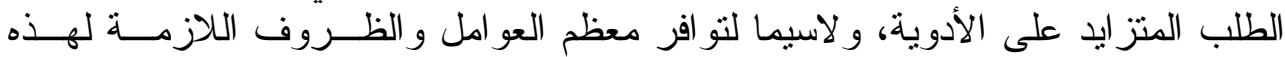

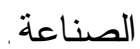

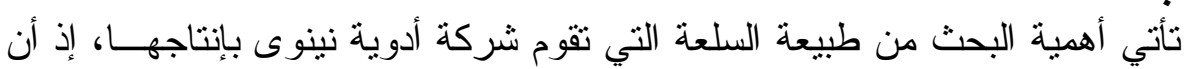

\section{أهمية البحث}

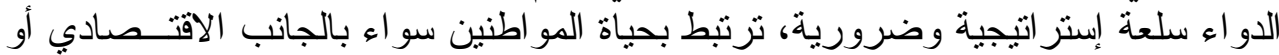

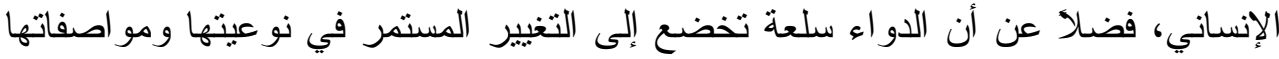

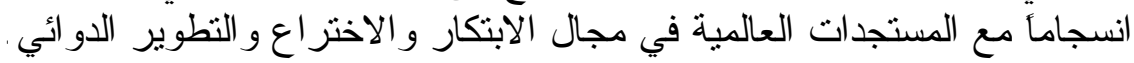

مشكلة البحث مث

تتمثل مشكلة البحث بعدم قدرة الشركة العامة لصناعة الأدوية في نينــوى (NDI)

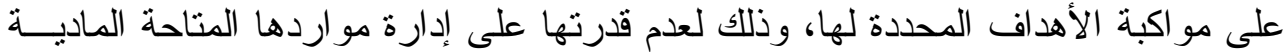

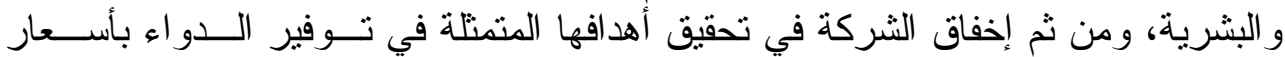

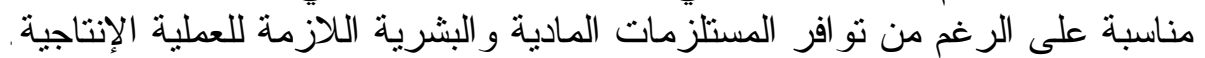

يهرف البحث إلى در اسة وتحليل و اقع الصناعات الدوائية في العر اق و إجر اء عملية

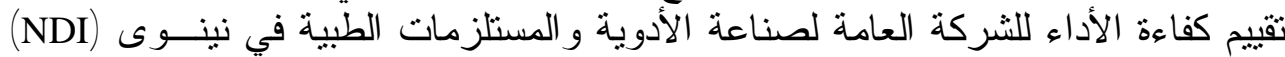

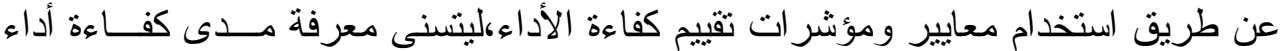

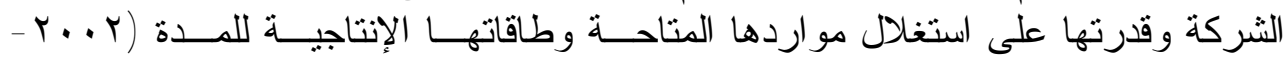

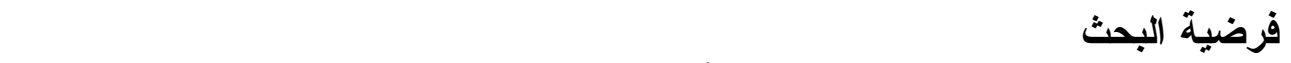

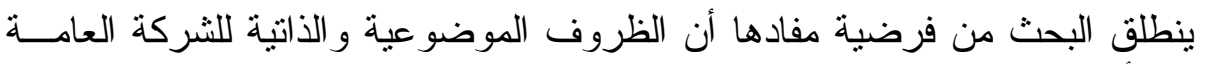

لصناعة الأدوية و المستلزمات الطبية في نينوى مواتية لاستغلال مو اردها المتاحة الماديــة المية 


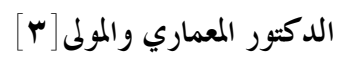

و البشرية وطاقاتها الإنتاجية، وأن معايير تقييم كفاءة الأداء تسهم في بيــان وتحديـــــاء أداء

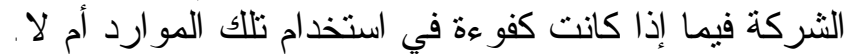

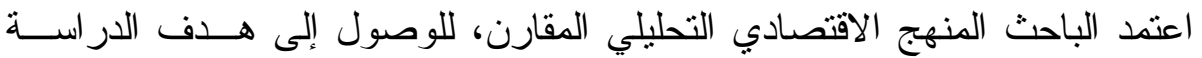

\section{منهج البحث البح}

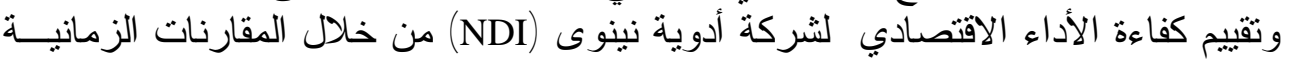

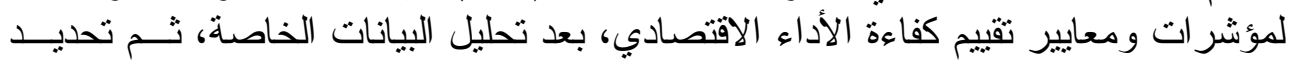

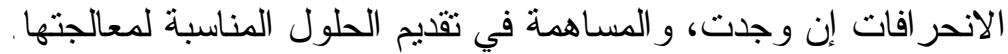

لقد ركز الجانب النظري على مجموعة من الفقرات ذات العلاقة بموضوع البحــث

$$
\text { أولاً - الجاتب النظري }
$$

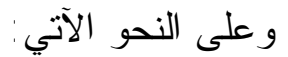
مفهوم تقييم كفاءة الأداء الاقتصادي وقد وصفت عملية تقييم الأداء بأنها قياس أداء أنشطة الوحدات الاقتصادية بادية بالاستتاد

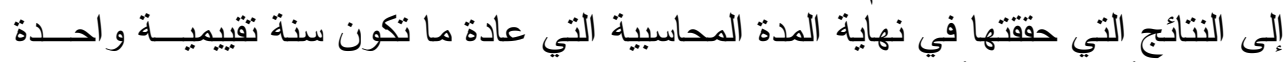

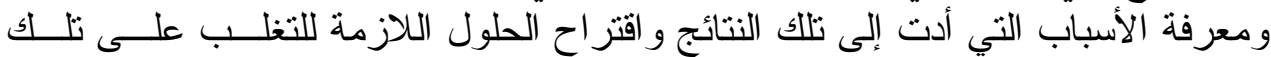

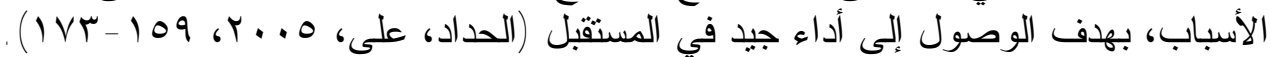

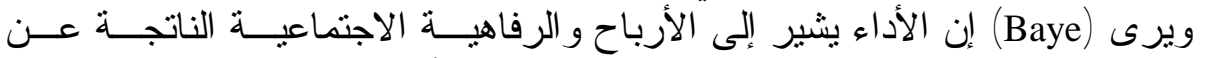

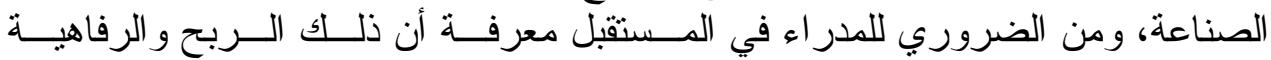

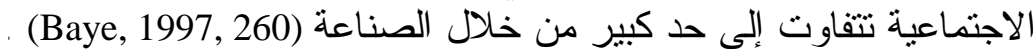

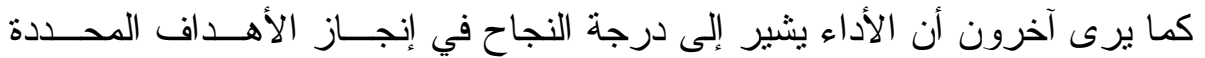

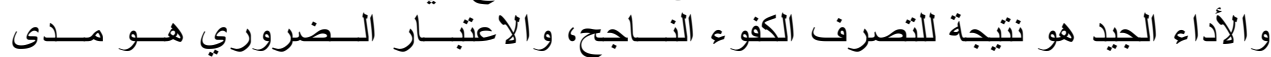

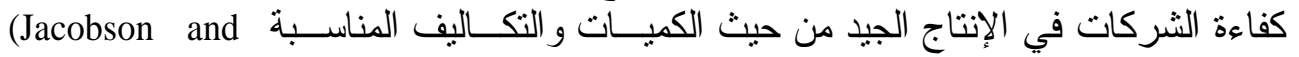
. Bernadette, 1996, 221)

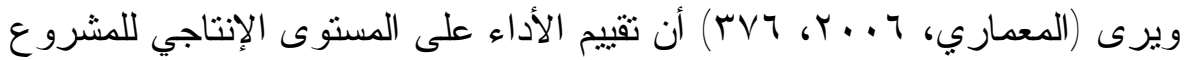

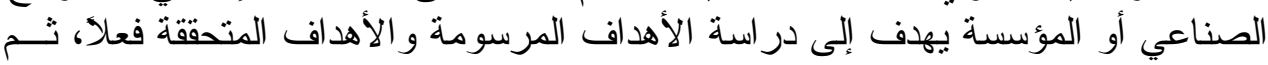

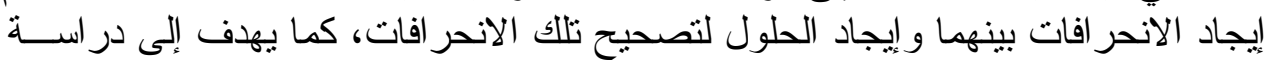

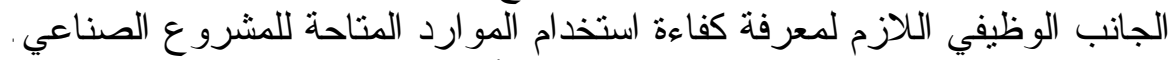

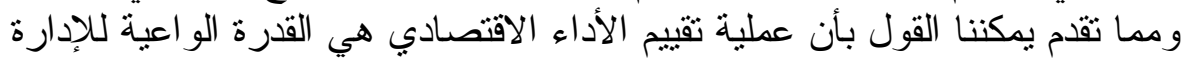

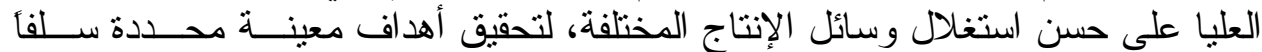

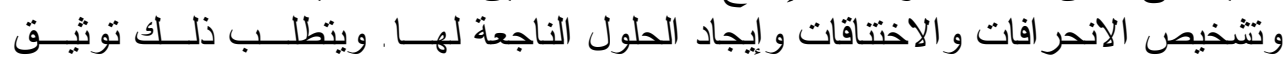

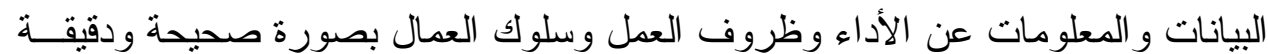

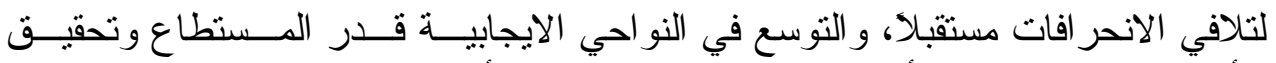

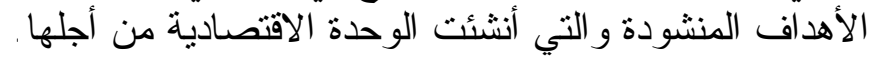

\section{The Importance of Economics Performance Evaluation}

\section{أهمية تقييم كفاعة الأداء الاقتصادي}

$$
\text { يمكن بيان أهية تقييم الأداء الاقتصادي في النواحي الآتية: }
$$




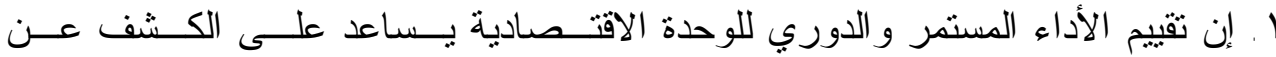

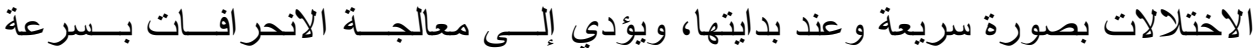

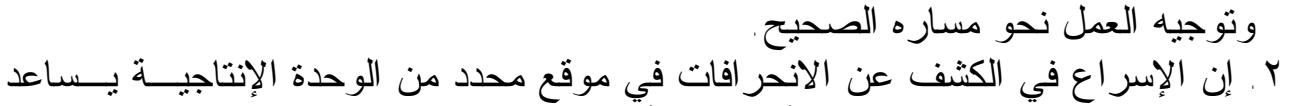
على عدم تسربها إلى المواقع الأخرى، الأمر الذي يؤدي إلى تحئ تحجيم الانحر الفات وتقليل

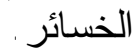

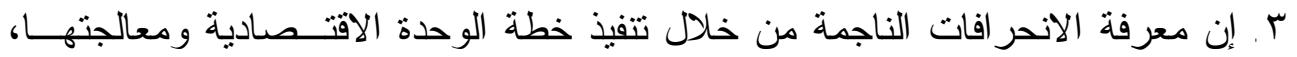

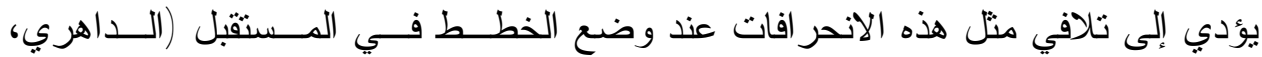
( ) أ.

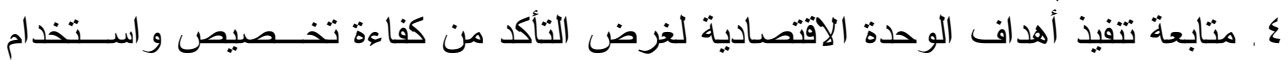
الموارد الإنتاجية على النحو الأفضل ولتحقيق الأهداف المحددة.

\section{Functions of Economics Performance Evaluation}

$$
\text { وظائف تقييم الأداء الاقتصادي }
$$

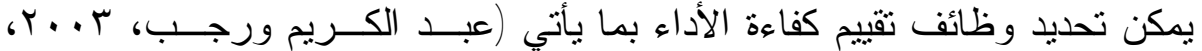

$$
\text { :( ) } 9 \text { 人- ) } 1 \vee 9
$$

ا ـ التعرف على مدى تحقق الأهداف المرسومة للوحدة الاقتصادية من خلال متابعة تتفيذ

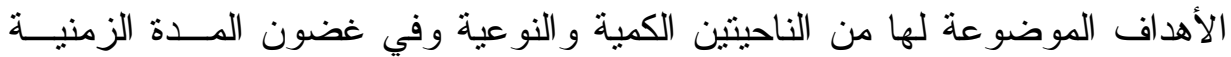
المحددة لها.

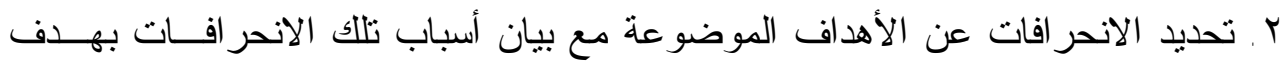
التعرف على كفاءة استخدام الموارد المادية و المالية و البشرية المانية المتاحة.

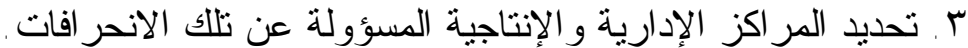

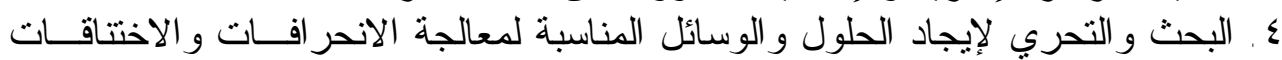
و الحيلولة دون وقو عهو لإيجاد في المستقبل.

\section{Goals of Economics Performance Evaluation}

$$
\text { أهداف تقييم الأداء الاقتصادي }
$$

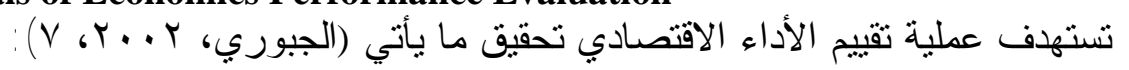

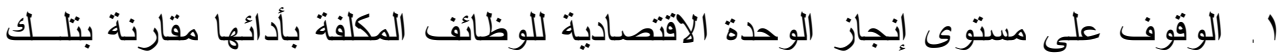

$$
\text { الوظائف المدرجة في خطنها الإنتاجية. }
$$

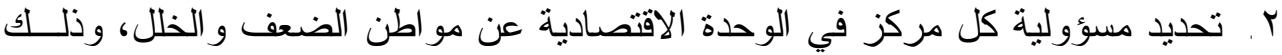

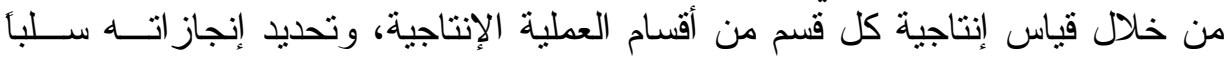

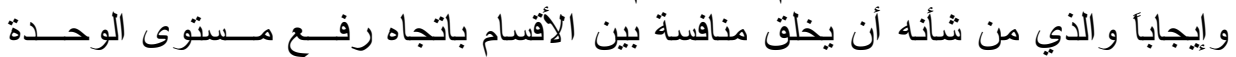
الاقتصادية و أدائها.

r. . مسـاعدة المستويات الإدارية على معرفة مدى الانحر افــات وبيــان أســبابها و اتخـــاذ

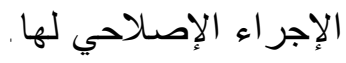
ع. ـ الوقوف على كفاءة استخدام الموارد المتاحة بطريقة رشيدة يحقق عائداً أكبر بتكــاليف 


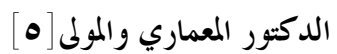

مراحل تقييم كفاءة الأداء الاقتصادي

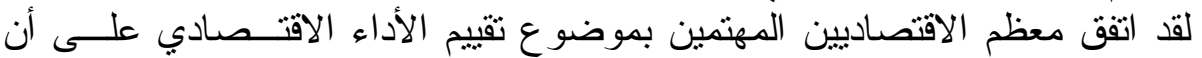

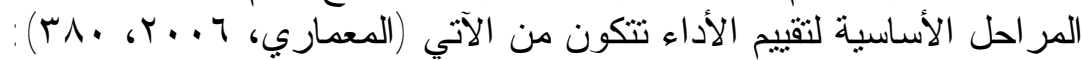

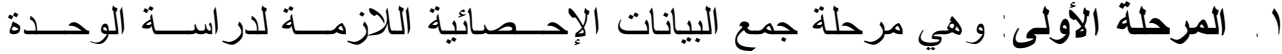

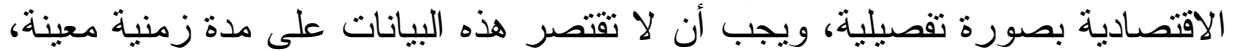

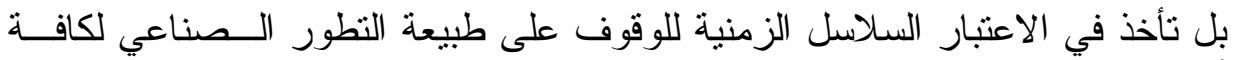

أهداف الوحدة الاقتصادية.

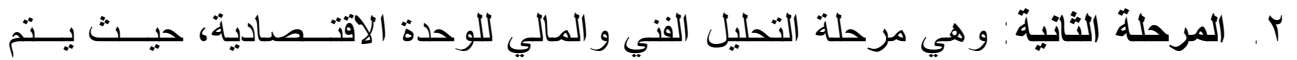

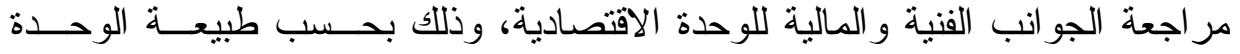

الاقتصادية.

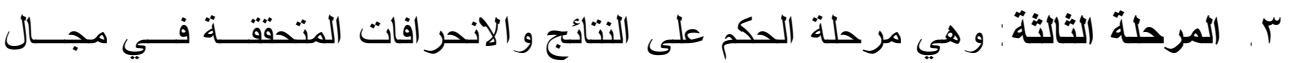

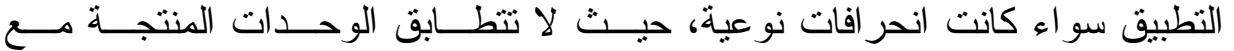

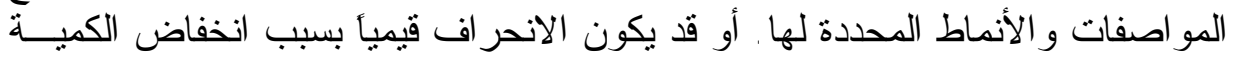
المنتجة، أو فنباً بسبب اختلال العلاقات الصناعية المتداخلة.

معايير تقييم الأداء الاقتصادي

تعد عملية تقييم الأداء مقياسأ لقدرة الوحدة الاقتصادية على تحقيق الأهداف المحددة

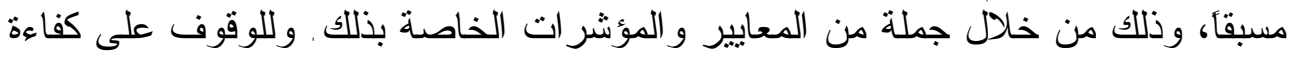

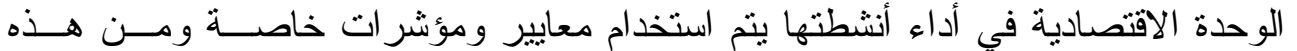
المعايير : الإندير

أولاً - معيار الإتتاجية Productivity Criterion

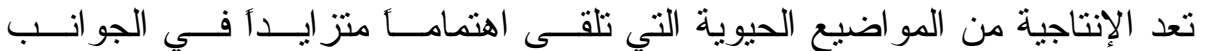

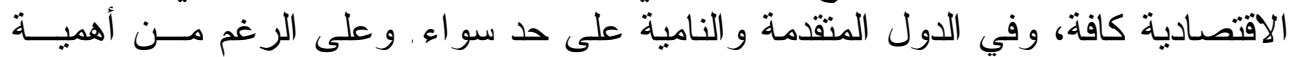

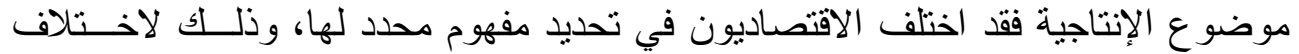

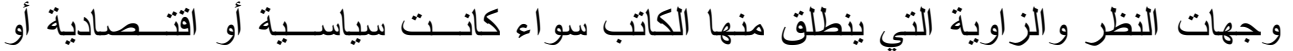
إدارية أو غير ها.

وقد ورد في مفهوم الإنتاجية (Productivity) بأنها قياس العلاقة بــين المخرجـات الكيات

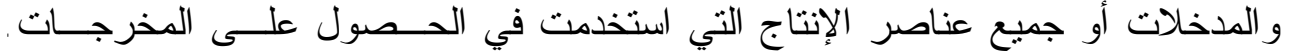

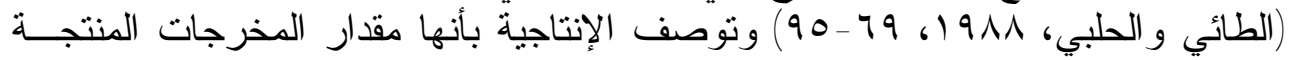

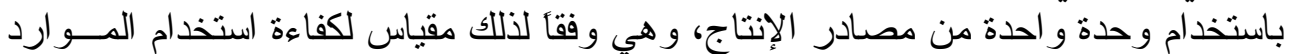

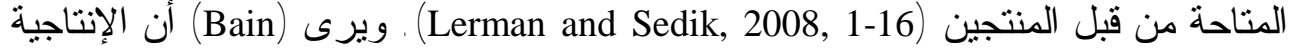

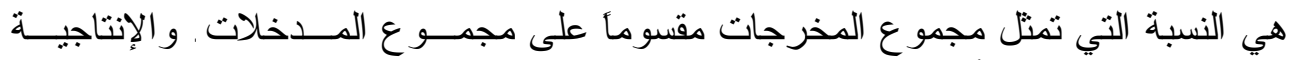

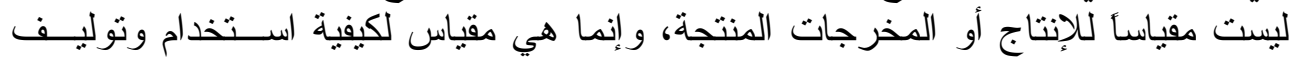
عناصر الإنتاج للحصول على نتائج مرغوبة (Bain, 1982, 3).

ثalue Added Criterion ثانياً - معيار القيمة المضافة

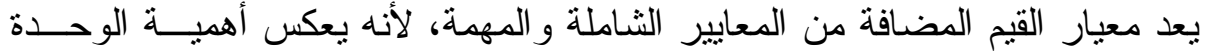

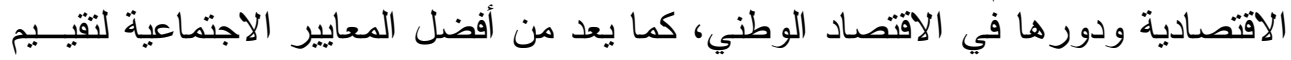




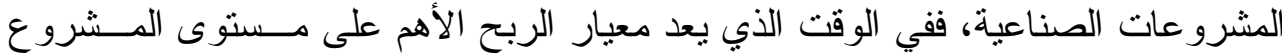

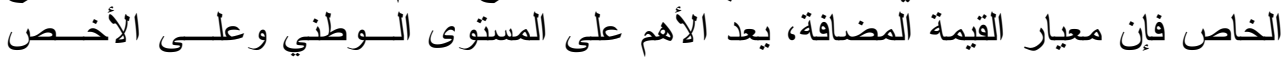

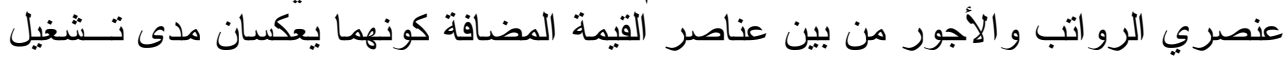

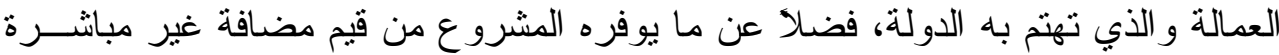

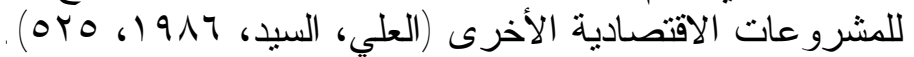

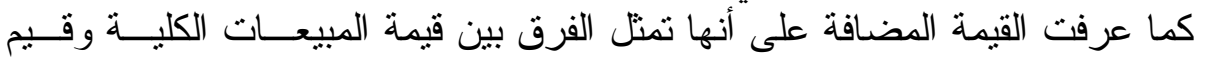

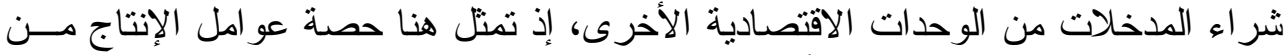

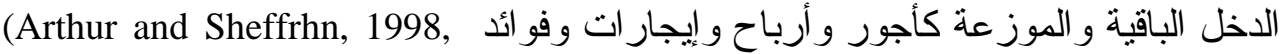

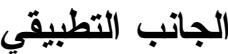 أولاً - التقييم باستخذام معيار الإتتاجية}

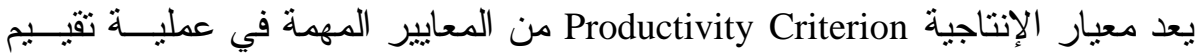

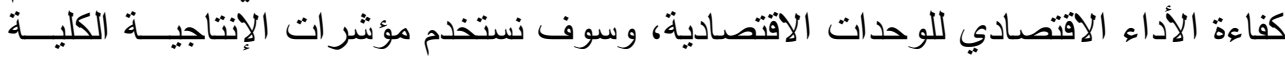

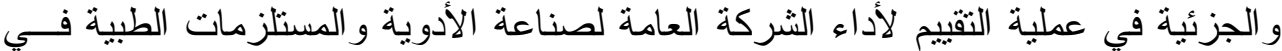

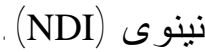

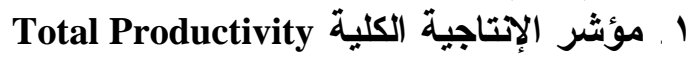

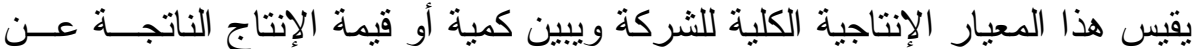

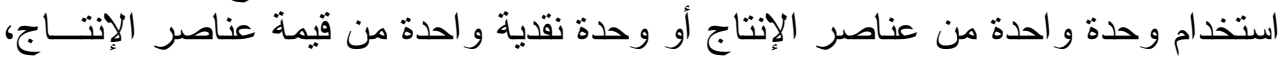

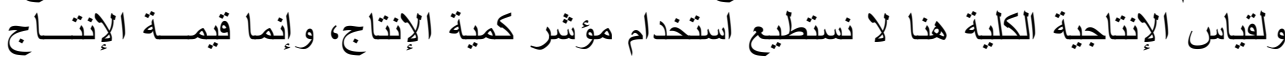

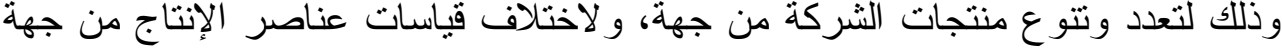

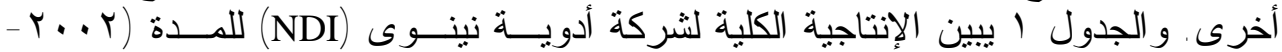
(r...

\section{الجدول الأوبة}

الإتاجية الكلية للثركة العامة لصناعة الأدوية و المستلزمات الطبية في نينوى

$$
\text { بالأسعار الجارية الألماية }
$$

\begin{tabular}{|c|c|c|c|c|c|c|}
\hline الإلإجلية & العناصوع & الأولية & رأس المال & العمل & قيمة الإتتاج & السنوات \\
\hline$r, 0$ & $r \cdot 1 \leqslant V \wedge$. & VqYo.. & rTort & $9.9 V \leqslant V$ & STYT & $r \ldots r$ \\
\hline 1,0 & $Y V T \leq V \backslash V$ & $\leq 19 \leqslant 99$ & $\varepsilon \ldots 19 r$ & 1910.10 & $r 99 \ldots 7$ & $r \ldots r$ \\
\hline $1, V$ & हVऽTrVV & $947 \leqslant 7 \leqslant$ & $\varepsilon \wedge 9911$ & & $\Lambda) \cdot r r \leqslant r$ & $r \ldots \varepsilon$ \\
\hline 1,0 & AY9197\& & YYVTY & 771070 & Oror10 & $T r \Lambda \cdot Y \ldots$ & $r \ldots o$ \\
\hline $1, \varepsilon$ & $|T| V \vee q \mid \varepsilon$ & 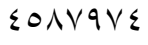 & 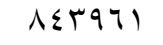 & $V V \leqslant 09 \vee 9$ & $1 \wedge \Lambda \vee \varepsilon \ldots$ & $r \ldots r$ \\
\hline 1,0 & $17 V 1991 \%$ & E79TKVV & IATOY & $1.194 Y 9 \varepsilon$ & TEYAY... & $r \ldots v$ \\
\hline
\end{tabular}

المصدر: الأعدة (1 -ـ) الثركة العامة لصناعة الأدوية و المستلزمات الطبية في نينوى اعقىم الحسابات العمود (0) من اعداد الباحث. 
الدكتور المعماري والمول

ويلاحظ من الجدول ا أن إنتاجية العناصر أو الإنتاجية الكلية بلغت (r,0) دينـار

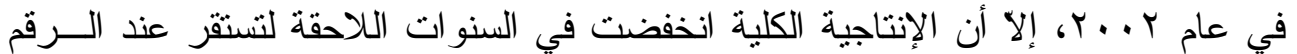

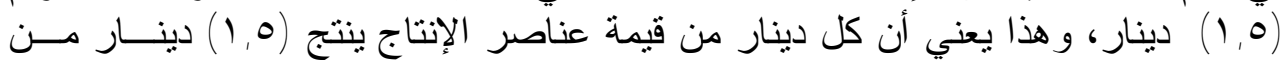

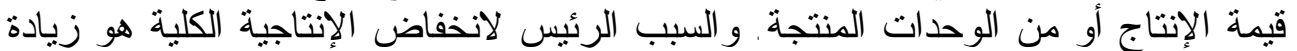

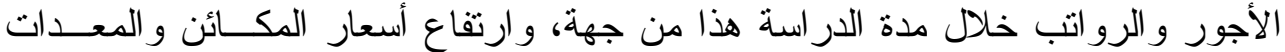

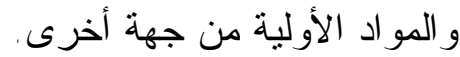

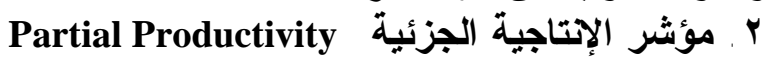

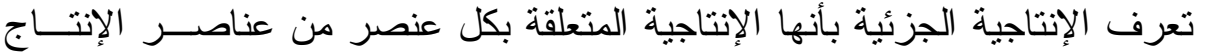

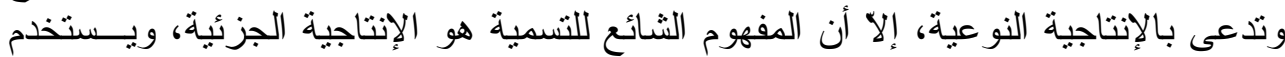

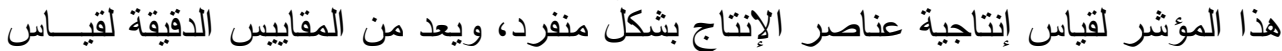

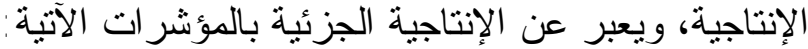

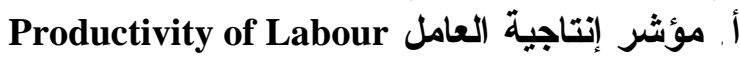

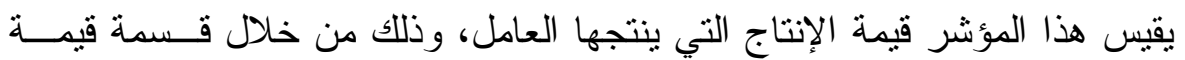

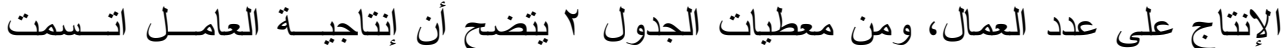

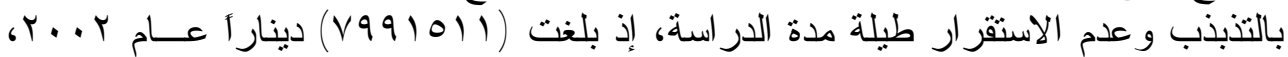

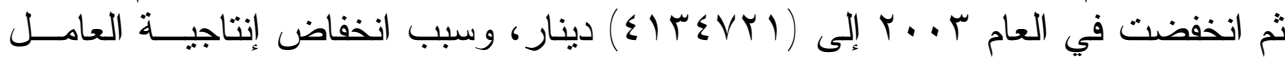

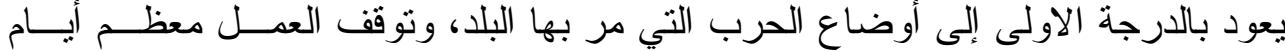

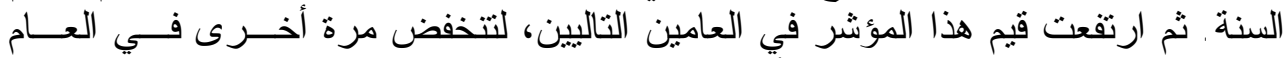

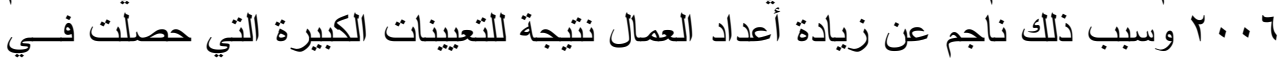

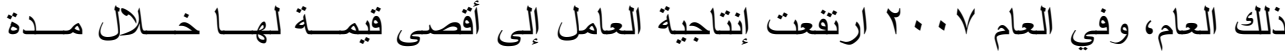

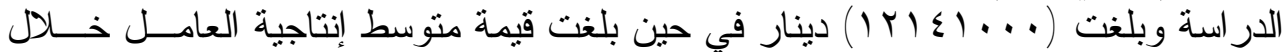

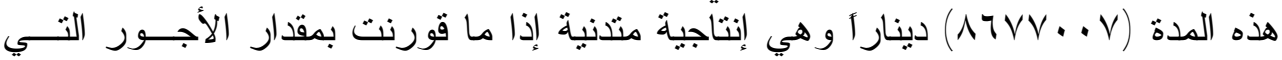

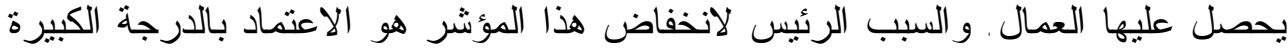

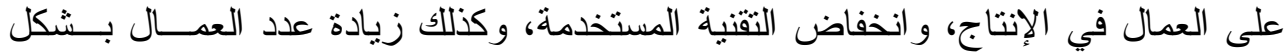

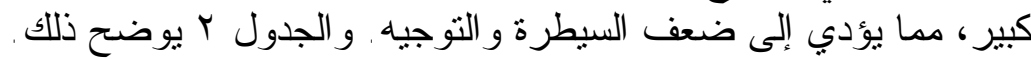

\begin{tabular}{|c|c|c|c|}
\hline \multicolumn{4}{|c|}{ 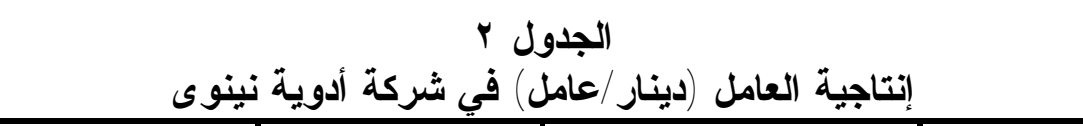 } \\
\hline 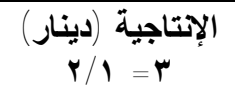 & عدد العمال & قيمة الإتتاج (ألف دينار) & السنوات \\
\hline 1991011 & 179 & $79 \leq \varepsilon 7 Y T$ & $r \ldots r$ \\
\hline$\sum|r \Sigma V Y|$ & 970 & $r q 9 \ldots 7$ & $r . . r$ \\
\hline$\lambda r \varepsilon \varepsilon r r q$ & $9 \times 1$ & $\Lambda 1 . r T \leqslant r$ & $r \ldots \varepsilon$ \\
\hline $11 \ldots \vee v \vee r q$ & $117 \pi$ & $T r \Lambda \cdot r \ldots$ & $r \ldots o$ \\
\hline $9 \wedge r \cdot r \mid r$ & $19 Y Y$ & 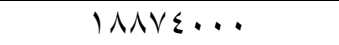 & $r \ldots r$ \\
\hline$T K \mid \leqslant 1 \ldots$ & $r \ldots$ & TEYAY... & $r \ldots V$ \\
\hline ATVV..V & $1 T \leqslant V$ & & المتوسط \\
\hline & باحث. & $(r, 1)$ & قصدم : الت الع \\
\hline
\end{tabular}


ب ب. إنتاجية المواد الأولية Productivity of Materials

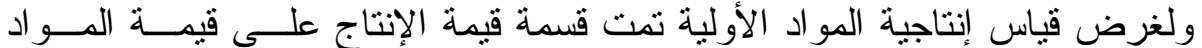

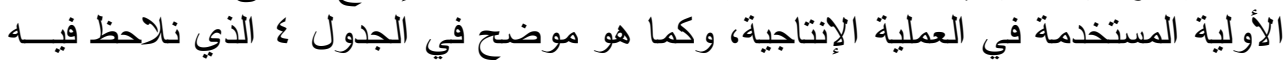

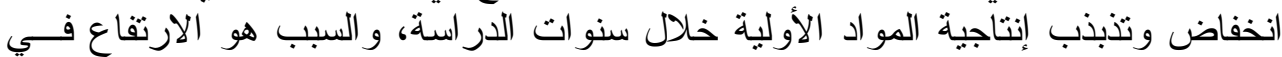

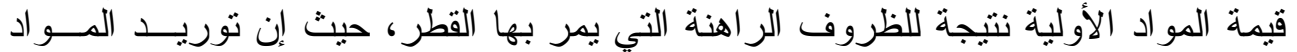

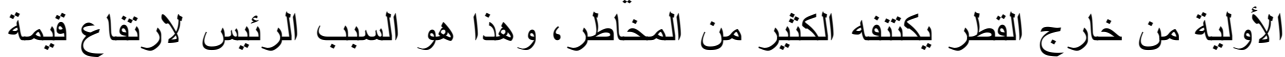

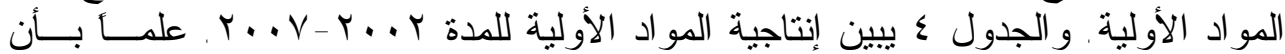

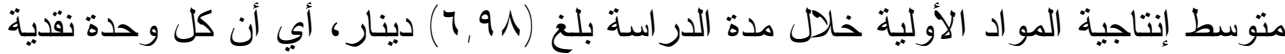

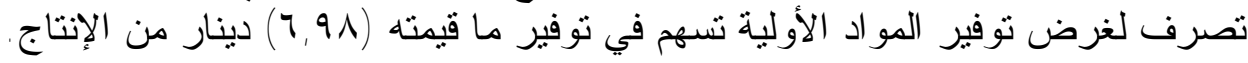

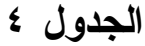

إنتاجية المواد الأولية في الشركة العامة لصناعة الأدوية في نينوى

\begin{tabular}{|c|c|c|c|}
\hline $\begin{array}{c}\text { (أنتاجية المواد الأولية } \\
\text { (الفينار) }\end{array}$ & قيمة المواد الأولية(ألف دينار) & قيمة الإتـاج (ألف دينار) & السنوات \\
\hline$\wedge, \wedge$ & VqYo.. & $79 \leqslant \leqslant T Y T$ & $r \ldots r$ \\
\hline 9,0 & $219 \leqslant 99$ & $r q 9 \ldots 7$ & $r \ldots r$ \\
\hline$\Lambda, V$ & 9 $4 \leq 7 \leq$ & $\lambda 1 \cdot r r \leqslant r$ & $r \ldots \varepsilon$ \\
\hline 0,7 & YYVTY & $\mid r \Lambda \cdot r \ldots$ & $r \ldots o$ \\
\hline$\varepsilon, 1$ & $\varepsilon 0 \wedge \vee 9 \vee \varepsilon$ & $1 \wedge \wedge \vee \varepsilon \ldots$ & $r \ldots T$ \\
\hline $0, r$ & ET9TrVV & TEYAY... & $r \ldots V$ \\
\hline 7,91 & YYA IVV & $1 Y \leqslant 9917 r$ & المتوسط \\
\hline
\end{tabular}

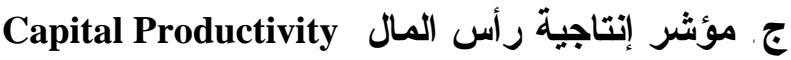

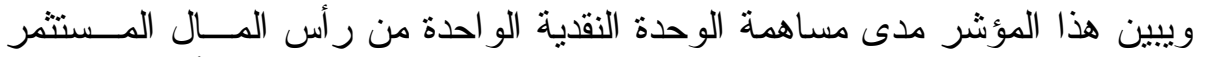

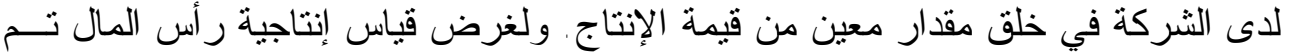

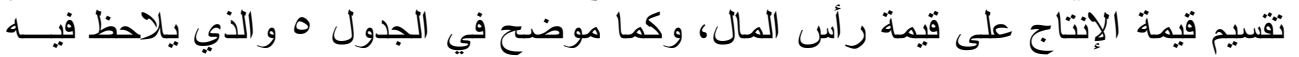

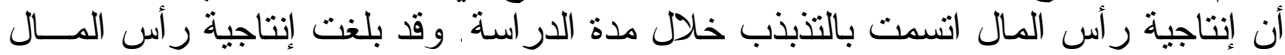

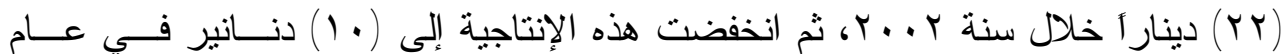

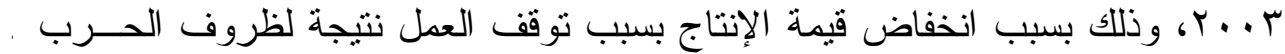

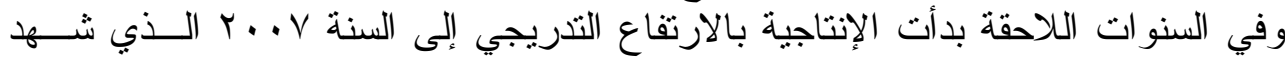

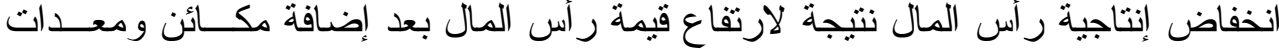
جديدة إلى الخطوط الإنتاجية في الثركة. 


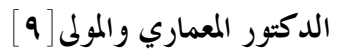

\begin{tabular}{|c|c|c|c|}
\hline \multicolumn{4}{|c|}{ 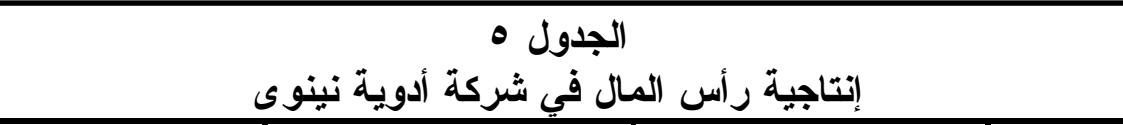 } \\
\hline 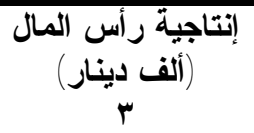 & قَيمة رأس المال (ألف & قيمة الإتتاج (ألف دينار) & السنوات \\
\hline rY & MTOKT & 79ะรTYT & $r \ldots r$ \\
\hline 1. & $\{\ldots 194$ & $r 99 \ldots 7$ & $r \ldots r$ \\
\hline IV & $\{\wedge 9911$ & $11 . r r \leqslant r$ & $r \ldots \varepsilon$ \\
\hline 19 & 774070 & IrA.r... & $r \ldots o$ \\
\hline rY & $\lambda \leqslant r q 71$ & 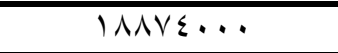 & $r \ldots T$ \\
\hline $1 \pi$ & MNOYEY & TEYAY... & $r \ldots v$ \\
\hline
\end{tabular}

ثانياً - التقييم باستخدام معيار القيمة المضافة

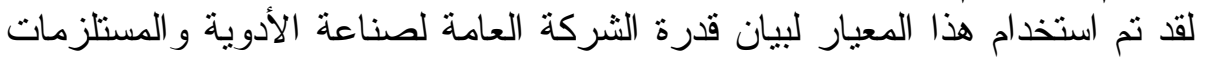

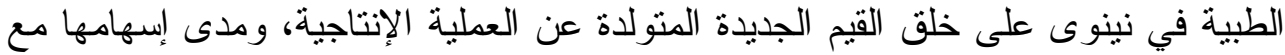

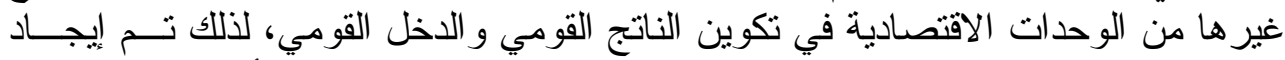

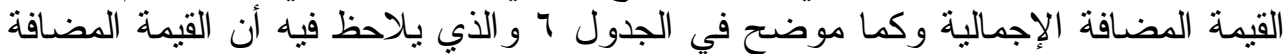

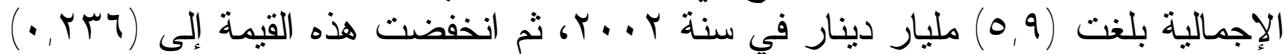

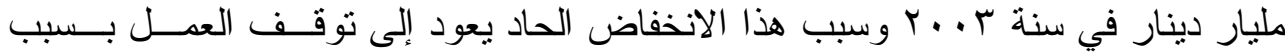

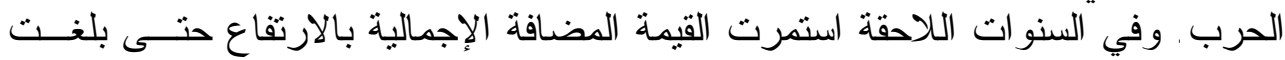

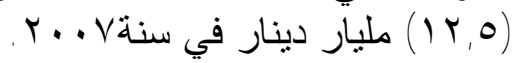

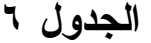

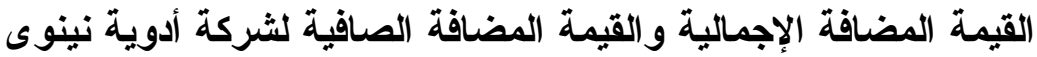

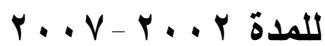

\begin{tabular}{|c|c|c|c|c|c|c|}
\hline 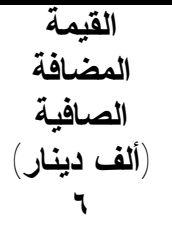 & الأدثار & 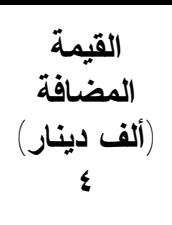 & الخديلزماتة & السليتية (ألف دينات & (ألف دينة الإتار) & السنوات \\
\hline 07r79rr & YTVVTV & $0 \wedge 9 \leq 79$. & rOVETr & varo.. & $79 \leq \varepsilon 7 r \pi$ & $r \ldots r$ \\
\hline $0 . Y V$ & $r M .7 .0$ & TrOTrT & ETIYYV & IIVYTEV & $r 99 \ldots 7$ & $r \ldots r$ \\
\hline$\varepsilon 001 \leqslant 1 \%$ & rᄉO०人० & ร947991 & $7.0 \leqslant .0$ & ro099E. & NI. rr & $r \ldots \varepsilon$ \\
\hline Tr.rVII & 071110 & $7 \vee \vee 1 \wedge 97$ & $71 Y \wedge 90$ & $0 \leqslant I V T \cdot 9$ & IrA.r... & 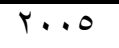 \\
\hline AY.TYIO & $V \vee 1199$ & 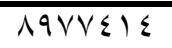 & $11.9 \leq 0 V$ & AVAVIYq & 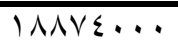 & $r \ldots T$ \\
\hline $11 \times 9.111$ & $|Y \cdot \Sigma Y M|$ & $1<\leqslant 9 \leqslant \varepsilon 19$ & $1 V \leqslant q r \cdot \Lambda$ & I.. rAYVK & rEイヘイ... & $r \ldots V$ \\
\hline
\end{tabular}


أولاً - الاستنتاجات

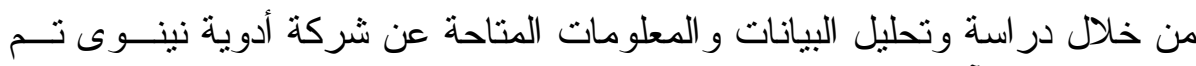

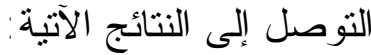
ا. انخفاض الإنتاجية الكلية وسبب ذلك يعود إلى ارتفاع قيمة الرو اتب و الأجــور نتيجــة

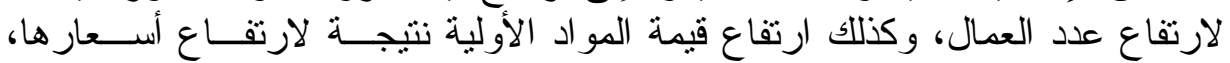

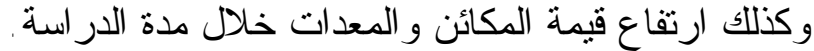

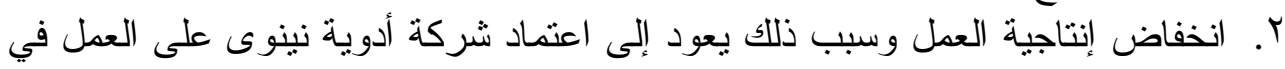

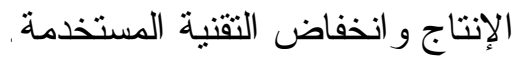

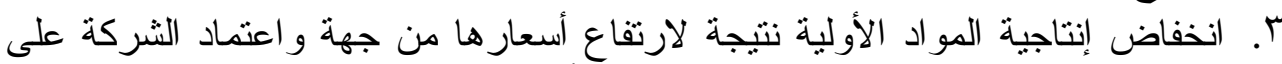

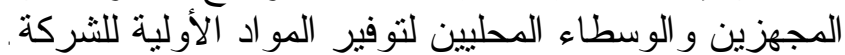
ء. ارتفاع إنتاجية رأس المال في الثركة، وهذاء يعود إلى انخفاض قيمة المكائن و الآلات المستخدمة في الإنتاج.

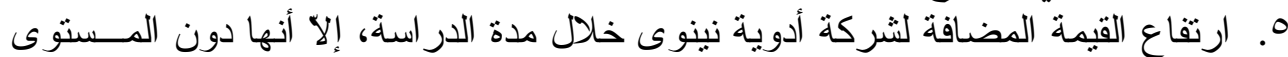

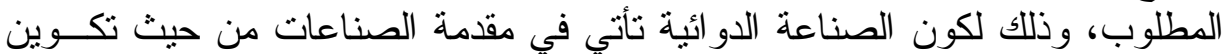

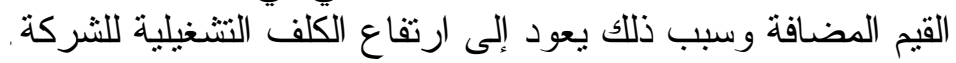

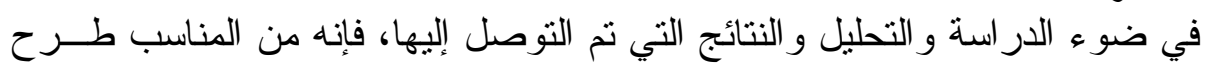
بعض المقترحات، و التي يمكن إيجاز ها بالاتي:

ا ـ التأكيد على ضرورة الاهتمام بعملية تقييم كفاءة الأداء الاقتصـادي في شــركة أدويـــة

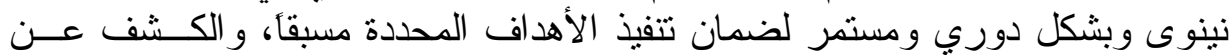

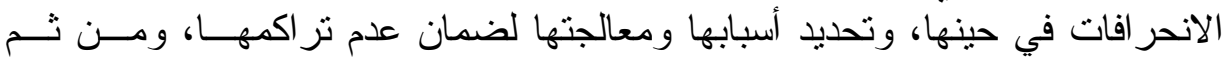
يصعب تحديدها و معالجتها مستقبلا.

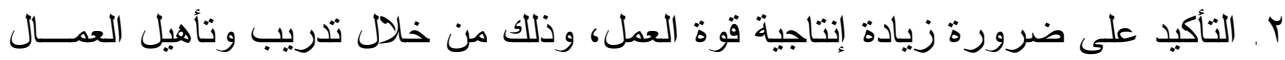

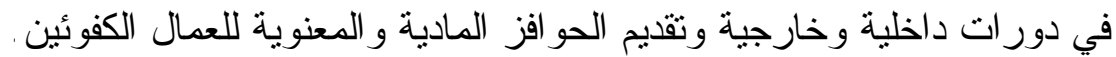
r. التأكيد على ضرورة قيام شركة أدوية نينوى بالاعتماد على التقنية المكثقة لر أس المال

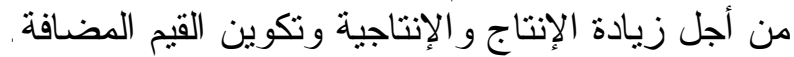

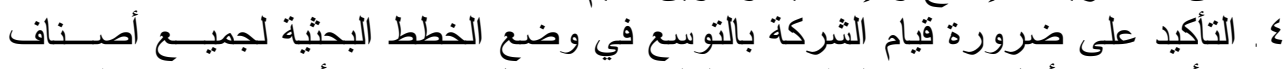

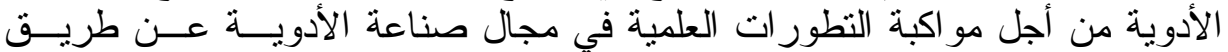

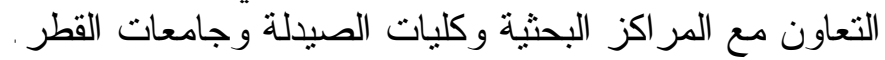

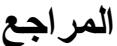 \\ أولاً - المر اجع باللغة العربية}

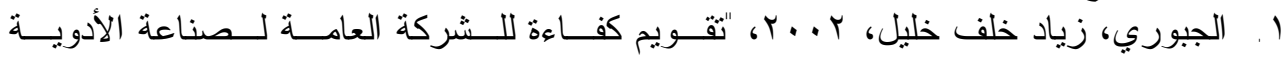

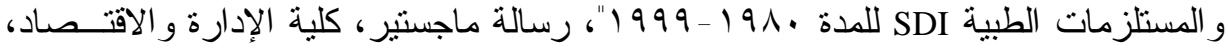


الدكتور المعماري والمولى[11]

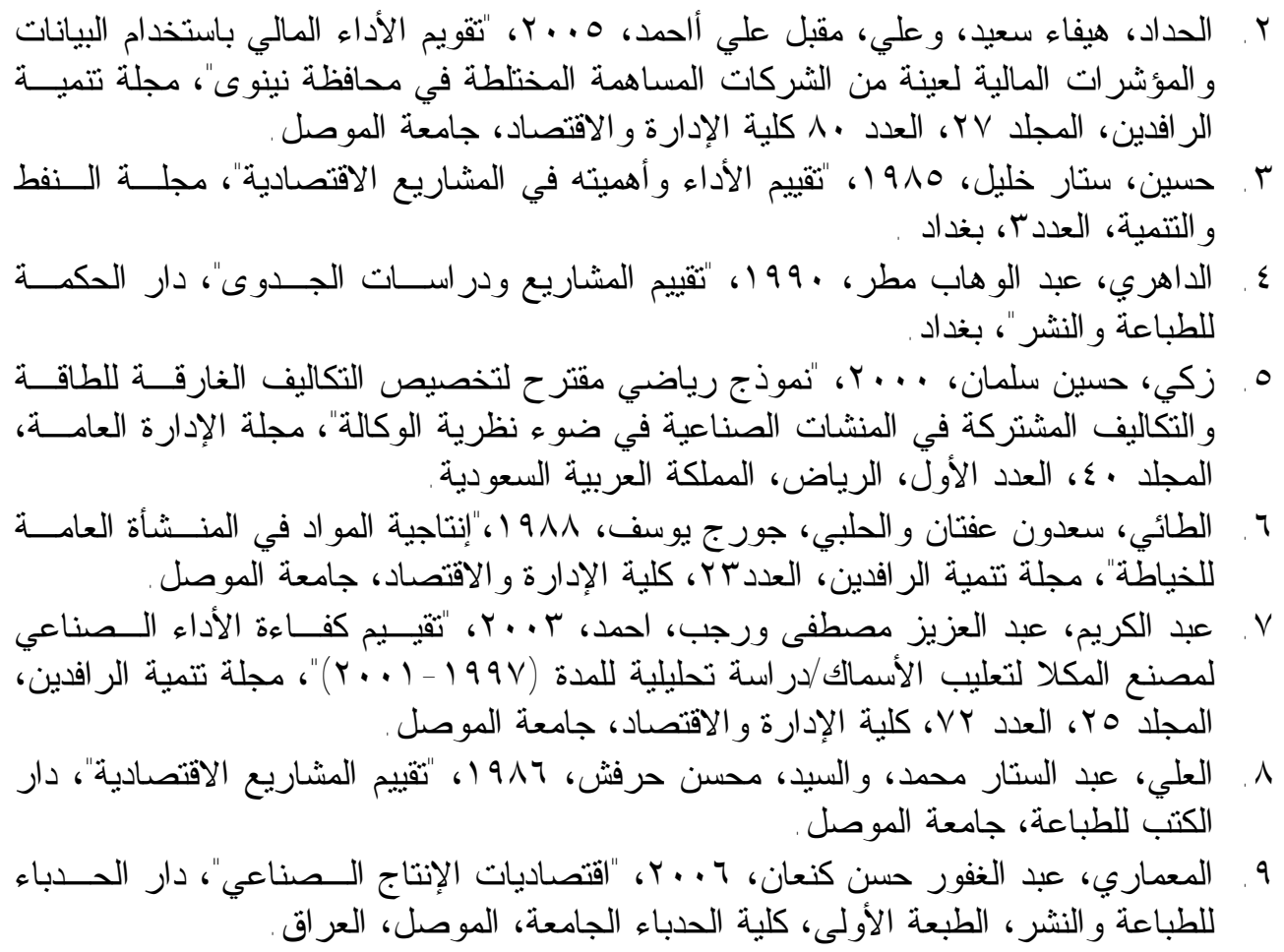

ثانياً - المر اجع باللغة الاجنبية

1. Arthur,O,Sullivan \& Sheffrin M Steven1998,"Macro Economics Principles And Tools ",Prentice-Hill.Inc, New jersey, U.S.A

2.Bain,David, 1982,"The Productivity Prescription the Managers Guide to Improving Productivity and Profits", McGraw-Hill Book Company, USA

3. Baye, Michael R .,1997,"Managerial Economics and Business Strategy", $2^{\text {nd }}$ ed, Irwin, USA.

4.Lerman,Zvi and Sedik, David.J 2008,"Productivity and Efficiency of corporation and Individual farms in UKRAINE".

http://departments.agri.huji.ac.il./economics/indexe.htmc.

5.Jacobson, David and O, Andresso-O, Callaghan, Bernadette, 1996,"Industrial Economics and Organization", McGra-Hill, Publishing Company, England. 